\title{
Patrones de estacionalidad de las especies de aves residentes y migratorias de los Pantanos de Villa, Lima, Perú
}

\section{Seasonal patterns of the resident and migratory bird species of the Villa Swamps, Lima, Peru}

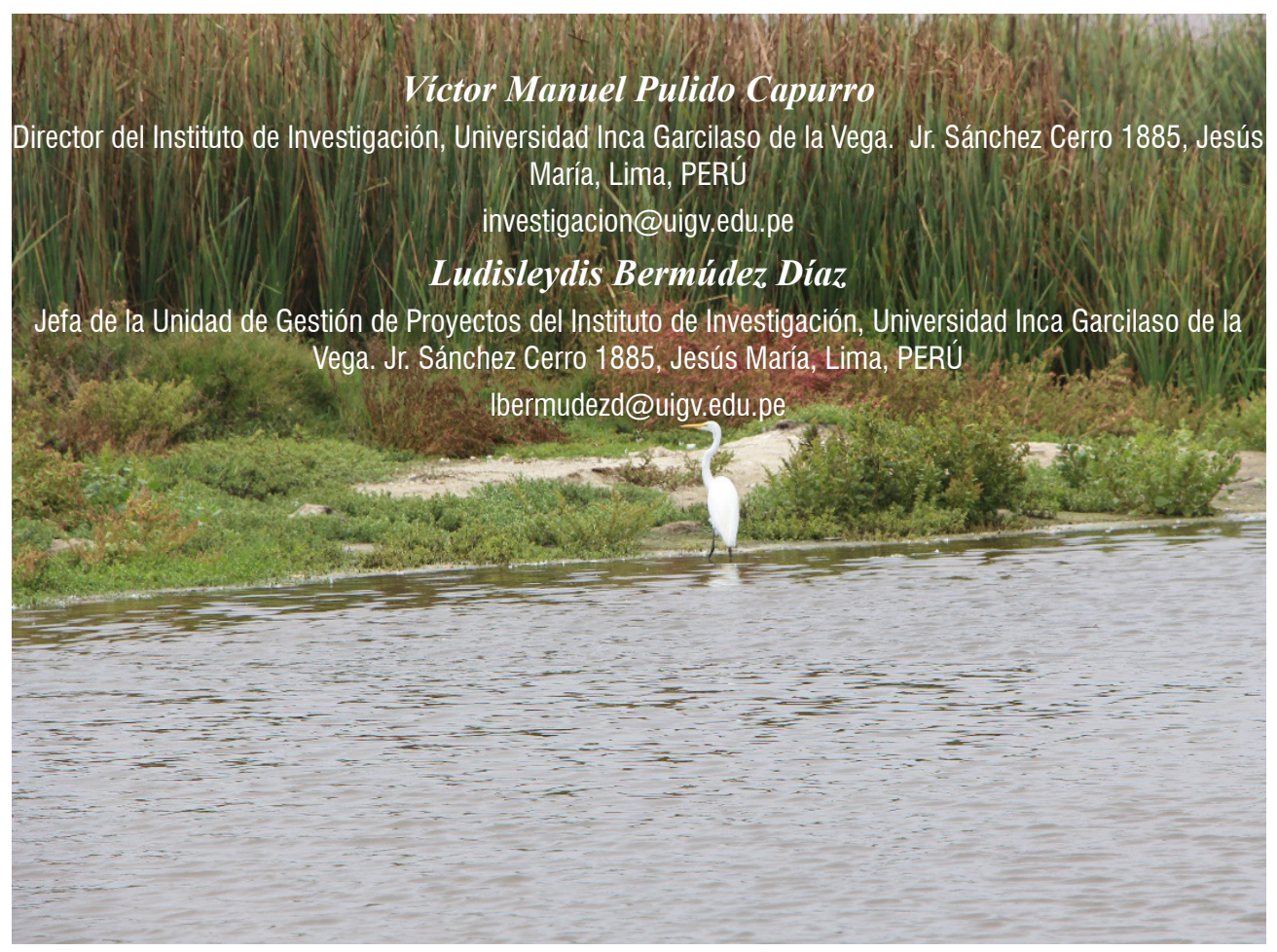


Recibido: Recibido: 20-VIII-2018; aceptado: 25-IX-2018; publicado online: 30-XI-2018; publicado impreso: 31-XII-2018

\section{Resumen}

En los Pantanos de Villa, Lima, Perú, se han registrado 211 especies de aves que se encuentran comprendidas en 145 géneros, 50 familias y 21 órdenes. Con respecto a la estacionalidad, 97 especies son residentes y 114 provienen de otras latitudes, que se dividen en tres grupos: las migratorias, las ocasionales y las introducidas. Las migratorias (82 especies) se diferencian en dos grupos: las que realizan migraciones horizontales, que proceden de la región Neártica (51 especies) y la región Austral (13 especies), y las migratorias verticales que realizan migraciones altitudinales y proceden de los Andes peruanos (18 especies); y las ocasionales (31 especies), que han sido registradas muy pocas veces y que, no siendo migratorias, se les ha reportado en los Pantanos de Villa. La migración de aves procedentes de la región Neártica tienen un patrón de variación estacional definido de setiembre a marzo. Y los registros de las migraciones de las especies provenientes de la región Austral y la región Altoandina evidencian que los patrones de estacionalidad de las mismas abarcan el periodo comprendido entre mayo y agosto.

Palabras clave: aves, Pantanos de Villa, migraciones de aves, patrones de variación.

\section{Abstract}

In the Villa Swamps, Lima, Peru, 211 species of birds have been registered and are included in 145 genera, 50 families and 21 orders. Regarding the seasonality, 97 species are residents and 114 are from other latitudes, which are divided into three groups: migratory, occasional and introduced. The migratory species (82 species) differ in two groups: those that carry out horizontal migrations, that come from the Nearctic Region (51 species) and the Austral Region (13 species), and the vertical migratory ones that make altitudinal migrations and come from the Peruvian Andes (18 species); and the occasional ones (31 species), which have been registered very rarely and which, although not migratory, have been reported in the Villa Swamps. The migration of birds from the Nearctic Region has a pattern of seasonal variation defined from September to March. And the records of the migrations of the species coming from the Austral Region and the Andean Highlands Region show that the seasonality patterns of them cover the period between May and August.

Keywords: birds, Villa Swamps, bird migration, patterns of variation.

Citación: Pulido, V. \& L. Bermúdez. 2018. Patrones de estacionalidad de las especies de aves residentes y migratorias de los Pantanos de Villa, Lima, Perú. Arnaldoa 25 (3): 1107-1128. DOI: http://doi.org/10.22497/arnaldoa.253.25318

\section{Introducción}

En los últimos sesenta años los Pantanos de Villa en Lima, Perú han venido sufriendo una fuerte presión antropogénica, por efecto de la ampliación de la frontera urbana, lo que ha ocasionado la reducción de importantes extensiones de hábitats de humedales, la disminución de las comunidades vegetales y la pérdida de parte de su diversidad biológica. (Pulido \& Bermúdez, 2018).

Las aves acuáticas migratorias son componentes sobresalientes de la biodiversidad global. Sus migraciones de larga distancia y su tendencia de concentrarse en grandes números en determinados humedales, las hace muy vulnerables porque dependen de una cadena de ambientes acuáticos altamente productivos para alimentarse y descansar, como parte de su migración anual (Bayly et al., 2018). Desde 1991, los Pantanos de Villa, forman parte de la lista de humedales de importancia internacional de la Convención Ramsar, basado en los criterios de: humedal representativo o único y específico basado en aves acuáticas (Ramsar, 2017); así como un área importante de conservación (IBAs) 
para las aves (Pronaturaleza, 2010; Amaro \& Goyoneche 2017; Senner \& Angulo, 2014).

Una gran diversidad de especies de aves acuáticas migratorias neotropicales y neárticas, en su mayoría especies de las familias Scolopacidae y Charadriidae, usan los humedales a lo largo de la ruta migratoria del Pacífico de América del Sur durante el período de migración (Torres, 2007). Estudios de poblaciones mediante técnicas de anillado indican que algunas aves playeras en Norteamérica dependen de áreas de descanso crítico durante sus migraciones hacia el norte y hacia el sur (Pulido et al., 1996). Otros estudios plantean que el $80 \%$ de algunas poblaciones de aves migratorias usan el área al mismo tiempo, las que pueden verse seriamente afectadas por la pérdida de estos sitios estratégicos (Butler et al., 1996). Las aves playeras se dispersan ampliamente en sus áreas reproductivas, ocupando los parches de hábitats apropiados para la nidificación y cría. Sin embargo, durante la migración y período no reproductivo se concentran en grandes números en determinados humedales costeros, aumentando su vulnerabilidad (García-Olaechea et al., 2018).

Las islas de la costa de Perú acogen millones de aves playeras, que cíclicamente se ven influenciadas por el Fenómeno de El Niño, ya que éste impacta sobre la abundancia de alimentos y diezma un porcentaje grande de las especies presentes (Garnica, 1999). Entre las aves marinas los pingüinos, piqueros, cormoranes, pelícanos, zambullidores, entre otros, encuentran sustento en los pocos humedales de las áreas desérticas (Pronaturaleza, 2010). El Perú es un país líder en el mundo en número de especies de aves con cerca de 1857 especies (Plenge, 2018). En este escenario, los Pantanos de Villa, ubicado en Lima resaltan como lugar de reproducción de numerosas especies (Amaro \& Goyoneche, 2017) así como apostadero y lugar de paso de aves migratorias, en su ruta de migración por el corredor a lo largo de la costa Pacífica de América del Sur (Pulido, 2018).

Aunque se han realizado diversos estudios sobre las aves residentes y migratorias que habitan en los Pantanos de Villa, los patrones de estacionalidad de las mismas no han sido debidamente corroborados. El objetivo del presente trabajo es establecer los patrones de estacionalidad de las especies de aves residentes y migratorias que habitan en los Pantanos de Villa, en Lima, Perú.

\section{Material y métodos}

Área de estudio: Los Pantanos de Villa están situados al sur de la ciudad de Lima, en el distrito de Chorrillos, provincia de Lima, en la intercuenca de los ríos Rímac y Lurín. Geográficamente se encuentra entre las coordenadas $12^{\circ} 10^{\prime}-12^{\circ} 13^{\prime} S$; $77^{\circ} 01^{\prime}$ $77^{\circ} 02^{\prime} \mathrm{W}$. El nivel de agua está asociado a las épocas de avenidas y estiajes del río Rímac. En las épocas de mayor caudal del río Surco (enero a marzo), se produce el riego de las áreas agrícolas y parques, dando lugar a mayor infiltración o recarga del acuífero con el consiguiente mayor afloramiento en Villa Baja. En épocas de estiaje, el comportamiento es todo lo contrario (SERNANP, 2016). La altitud varía desde el nivel del mar hasta los $5 \mathrm{~m}$, siendo la profundidad máxima de 1,5 $\mathrm{m}$ en los espejos de agua. La precipitación total mensual promedio oscila entre 0,0 $\mathrm{mm}$ y $5,5 \mathrm{~mm}$. La temperatura media mensual presenta una variación moderada a lo largo del año con valores máximos en marzo con $25,8{ }^{\circ} \mathrm{C}$ y $15,6^{\circ} \mathrm{C}$ en setiembre. La humedad relativa media es de $86 \%$ y la evaporación oscila entre $30 \mathrm{~mm} / \mathrm{mes}$, de junio a setiembre y $225 \mathrm{~mm} / \mathrm{mes}$, de enero a marzo. Los valores máximos de horas 
de sol son de 9 horas y los mínimos de 0,5 horas. La nubosidad promedio anual es de 4 octavios (Young, 1998).

Descripción de hábitats: Las zonas hidromórficas, constituyen la unidad geomorfológica central, compuesta por lagunas irregulares, islas, canales, juncales, turberas, salitrales y gramadales que se interrelacionan en forma gradual y temporal en función de las estaciones climáticas y fenómenos excepcionales de precipitaciones o sequías. Los límites entre una comunidad y otra no están estrictamente definidos, debido a las variaciones de los niveles de agua así como a los procesos de sucesión vegetal los cuales traen como consecuencia cambios en la composición taxonómica de las comunidades. Los hábitats presentes en los Pantanos de Villa son los siguientes (Pulido \& Bermúdez, 2018).

Laguna con espejo de agua. Conformado por la laguna y los canales que discurren en el interior de los pantanos; de 0,5 a 1,5 $\mathrm{m}$ de profundidad y de 50 a $10000 \mathrm{~m} 2$ de superficie; aguas pardo claras hasta turbias, de salinidad variable.

Totoral. En suelos inundados o en los bordes de los espejos de agua; caracterizado por comunidades densas de totora (Typha domingensis).

Zona arbustiva. Formada por arbustos y cañaveral ubicados entre el totoral y vega de Ciperaceas, cerca al espejo mayor de agua; matorral denso dominado por especies leñosas y arbustivas de dos a cinco metros de alto.

Vega de ciperáceas. En suelos de substratos inundados, cerca de cuerpos de agua, totoral o gramadal; plantas de 0.5 a $1 \mathrm{~m}$ de alto, especialmente Schoenoplectus americanus y Paspalum vaginatum.

Gramadales. Abarca la mayor superficie sobre suelos arenosos con presencia de Distichlis spicata, Paspalum vaginatum, Schoenoplectus americanus y Sporobolus virginicus.

Canales y depresiones. Tienen de uno a dos metros de ancho y de 0,5 a dos metros de profundidad; controlan el flujo de agua o sirven como drenes, sus aguas son dulces ( 0 a $5 \%$ de salinidad) y relativamente claras.

Litoral marino. Las corrientes marinas locales y la acción de las olas y el viento, deposita cúmulos de arena que son la interfase entre el mar y la planicie de Villa.

Parques y jardines. Presencia de plantas exóticas, de fuerte influencia antrópica, con características especiales relacionada con zonas arbóreas y arbustivas.

Identificación de especies. Se realizó el registro de las especies de aves desde el año 1973 hasta el 2018, en los hábitats previamente establecidos por Young (1998) y Pulido \& Bermúdez (2018). Para las observaciones de aves se utilizaron binoculares $(7 \times 35)$ y las Guías de campo de Koepcke (1964), Schulenberg et al. (2010) "Aves de Perú", y para la clasificación taxonómica se siguió a Plenge (2018), en "List of the birds of Peru". Esta información fue complementada mediante registros históricos, para lo cual se recurrió a las fuentes de información bibliográfica; respecto a la variación estacional de las especies, evidencias de aves en proceso reproductivo y en proceso de migración.

\section{Resultados}

Para los Pantanos de Villa se registran 211 especies, de las cuales 97 son residentes y 114 provienen de otras latitudes (Tabla 1).

Especies residentes. Las 97 especies residentes, permanecen durante todo el año en Pantanos de Villa (Fig. 1); 61 de 
ellas se reproducen en Villa (Fig. 2) y 36 de ellas aunque residentes en los Pantanos se reproducen en otras áreas vecinas de la costa peruana. Estas áreas son algunos parques urbanos de la ciudad de Lima, la zona desértica de Conchán, islas cercanas al litoral de Villa, desembocadura del río Lurín y las zonas donde están situadas las pozas de oxidación, que están rodeadas de áreas de cultivo y bosques, en los distritos de San Juan de Miraflores y Villa el Salvador. Las aves marinas costeras como los "pelícanos", "piqueros", "gaviotines", y "cormoranes" anidan en grandes colonias en pocas localidades y durante el periodo de la postcría, se dispersan a lo largo de la costa. Los humedales a lo largo del Pacífico, como la Albufera del Paraíso y los humedales de Ventanilla al norte de los Pantanos de Villa, así como los humedales de Puerto Viejo al sur de los Pantanos de Villa, albergan grandes concentraciones de las diferentes especies de aves acuáticas.

Especies procedentes de otras latitudes. Son 114 especies de aves procedentes de otras latitudes que habitan en los Pantanos de Villa y se dividen en tres grupos: las migratorias, las ocasionales y las introducidas. Las migratorias (82 especies) se diferencian en dos grupos: las que realizan migraciones horizontales; que proceden de la Región Neártica (51 especies) y la Región Austral (13 especies) y las migratorias verticales que realizan migraciones altitudinales y proceden de los Andes peruanos (18 especies). Ocasionales (31 especies), aquellas especies que han sido registradas muy pocas veces o excepcionalmente, y que no siendo migratorias, se les ha reportado en los Pantanos de Villa, que no corresponde a su área de distribución, o que han sido registradas en años en que se ha producido el fenómeno de "El Niño". Y una especie, Anas platyrhynchos, introducida en
1994, aunque actualmente ya no se observa ningún ejemplar.

\section{Distribución estacional de las aves que habitan en los Pantanos de Villa}

Los registros históricos bimensuales de las aves residentes y migratorias que habitan en los Pantanos de Villa, ha permitido establecer que las aves residentes, ya sea que se reproduzcan o no, son observadas durante todo el año (Fig. 3). Por otro lado, las aves migratorias Neárticas sólo son registradas en enero, marzo, setiembre y noviembre, lo que indica que el patrón de migración comprende el periodo setiembremarzo de cada año (Fig. 4). Respecto a las aves migratorias provenientes de la región Austral, si bien se han registrado durante todo el año, se observan con mayor frecuencia en los meses de mayo y julio. Las aves migratorias andinas se registran en enero, mayo, julio, setiembre y noviembre. Si bien no se registra un patrón definido, se observó que en mayo y julio están presentes en mayor número de especies y con menor frecuencia en setiembre. Las aves visitantes ocasionales se han observado en enero, marzo, julio, setiembre y noviembre, registrándose la mayor cantidad de especies en enero, marzo y noviembre.

\section{Discusión}

La migración en las aves es un proceso complejo que requiere la concurrencia de una serie de factores como la duración de los desplazamientos migratorios, cambios fisiológicos para el almacenamiento de nutrientes que utilizan durante el vuelo y adaptaciones para la navegación y orientación (Fuller \& Wainwright, 2018). La supervivencia de las poblaciones de las especies migratorias depende de la manera en que enfrenten los riesgos en sus áreas de reproducción, en las de invernada 
y durante el viaje entre ambos lugares (Bayly et al., 2018). Esta característica de las aves migratorias las hace vulnerables a las variaciones ambientales y a los cambios producidos por las actividades antropogénicas (McKinnon \& Love, 2018).

La disparidad entre las extensas áreas de cría y el reducido número de humedales utilizados para la alimentación y descanso durante la migración y período no reproductivo, conducen a enormes concentraciones de aves playeras en pequeñas áreas. Decenas y hasta cientos de miles de individuos, a veces constituyen hasta el 70 u $80 \%$ de la población total de una especie, pueden depender de un sólo humedal en determinado período del año (García-Moreno et al., 2007). En el caso de que alguno de estos sitios se viera afectado en cuanto a cantidad y/o calidad de hábitats por la contaminación, el drenaje, la expansión urbana o el turismo no regulado y últimamente por efecto de los cambios climáticos, poblaciones enteras de estas aves podrían verse seriamente amenazadas (Rakhimberdiev et al., 2018). El manejo inadecuado del agua puede originar la pérdida de la zona de limo poniendo en riesgo a las aves migratorias que hacen uso de este lugar, las cuales pueden ser consideradas aves emblemáticas de este tipo de hábitat (Torres et al., 2006).

Las aves acuáticas migratorias utilizan una variedad de humedales a lo largo de la ruta desde Canadá, Estados Unidos de Norte América, México, Centroamérica y el Pacífico sur y las costas de Chile, incluyendo manglares, estuarios, pantanos de agua dulce, marismas, lagos, albuferas y salares, siendo de gran importancia económica para las poblaciones locales (Delany, 2002). A lo largo de esta ruta de migración, la mayor parte de los hábitats están conservadas y algunos, todavía, no cuentan con mayores presiones antropogénicas. El litoral peruano de la costa del Pacífico comprende 3,080 km (Pulido et al., 1996); y en este escenario, históricamente los Pantanos de Villa constituyen, uno de los lugares más importantes de alimentación y refugio, en la ruta de numerosas especies migratorias provenientes de la Región Neártica, Región Austral, de los Andes peruanos y de otras latitudes (Morrison \& Ross, 1989).

Se distinguen dos etapas bien definidas una de setiembre a marzo, caracterizada por el notable incremento de especies migratorias neárticas, y la otra de abril a agosto cuando las migratorias altoandinas, las de la Región Austral y las residentes constituyen el grueso de la población. Una característica importante es la sincronización en el proceso de la migración (Gomez et al., 2017). La migración de aves procedentes de la Región Neártica o migratorias horizontales, presentan un patrón de variación estacional definido. De setiembre a marzo el número de especies migratorias es mayor que en los meses de mayo, junio, julio y agosto (Iannacone et al., 2010).

Durante todo el año, hay una población estable y residente, sumado a ella hay una población fluctuante que de setiembre a marzo está constituida por las migratorias neárticas (Fig. 5) y una vez que estas han regresado al hemisferio norte inmediatamente es ocupada por las migratorias australes y andinas (GarcíaOlaechea et al., 2018). La variación anual de la abundancia de individuos presenta una marcada regularidad determinada por la precisión en la estacionalidad de las migraciones de aves provenientes de diferentes latitudes (Fuller \& Wainwright, 2018). Rappole (2013) indica que conforme se llega más hacia el sur del Hemisferio Occidental las migratorias neárticas 
disminuyen tanto en número de especies como de individuos.

Las migratorias neárticas visitan todos los hábitats de la región (Fig. 6) pero una diversa cantidad de ellas llega a las áreas barrosas de las lagunas costeras, donde no encuentran competencia de las especies nativas a excepción de Charadrius vociferus y Charadrius alexandrinus (Pulido, 1998). Los hábitats a lo largo de la costa del Pacífico son importantes para las poblaciones de aves playeras neárticas, como Calidris alba, Limosa haemastica, Tringa melanoleuca, Tringa flavipes, Numenius phaeopus, Catoptrophorus semipalmatus y Calidris mauri (Schmidt et al., 1999; Iverson et al., 1996). Así, se tiene que, en Villa se han registrado 33 de las 36 aves playeras migratorias neárticas que pasan por todo Colombia (OcampoPeñuela, 2010). En un estudio efectuado por Osorio-Olarte (2012), durante 60 años en Bogotá (1945-2005) presentó una lista de 45 aves migratorias que siguen la ruta de la costa Pacífica de las cuales 15, llegan a Villa, entre octubre y mayo: Actitis macularia, Anas discors, Bartramia longicauda, Buteo platypterus, Calidris melanotos, Falco peregrinus, Hirundo rustica, Leucophaeus atricilla, Pandion haliaetus, Porzana carolina, Riparia riparia, Tringa flavipes, Tringa melanoleuca, Tringa solitaria, Tyrannus tyrannus.

La dinámica de la migración indica que no todas las aves llegan o parten al mismo tiempo. Pero que cada vez se ve mayormente influenciada por los cambios climáticos que suceden en Canadá y Estados unidos de Norte América (Galbraith et al., 2014). Cada año el paso de las aves migratorias neárticas por Villa empieza a inicios de setiembre, incrementándose en octubre y noviembre, en tanto en diciembre y enero presentan un periodo relativamente quieto, aumentando las poblaciones en febrero e incrementándose notablemente en marzo, y declinando rápidamente en mayo cuando se empieza a observar el cambio de plumaje de invernada por el de reproducción (Pulido, 2018; Podestá et al., 2017). Algunas especies como Calidris melanotos, Calidris bairdii y Phalaropus tricolor, ocurren primariamente durante los dos periodos de paso con un intervalo entre ellas (Cruz et al., 2007). Otras como Calidris alba, Tringa flavipes, Tringa melanoleuca, Leucophaeus pipixcan y Sterna hirundo son tan abundantes en el periodo de residencia como en los periodos de paso tendiendo sus poblaciones a incrementarse durante estos últimos. Algunas especies aparecen solo en uno de los dos periodos como Pluvialis dominica, Calidris himantopus, Sterna sandvicensis, que pasan entre febrero y abril, y otras como Aphriza virgata, Catoptrophorus semipalmatus que empiezan concentrándose entre setiembre y diciembre inician el retorno al norte (Senner \& Angulo, 2014). Normalmente las aves migratorias neárticas están ausentes en junio, julio y agosto a excepción de algunos individuos que por alguna razón no han retornado a sus lugares de anidamiento para completar su ciclo reproductivo (Torres et al., 2006). Este mismo patrón observado para Villa se repite para las Lagunas de Mejía en el departamento de Arequipa (Hughes, 1991; Pulido, 1987), en los humedales de Ventanilla y Puerto Viejo (Torres, 2007) y en los humedales de Eten (Angulo et al., 2010), en la laguna el Paraíso (Cruz et al., 2007).

De acuerdo a los registros históricos (Pulido, 2018), enero es el mes en que se reporta el mayor número de especies (160), el cual desciende ligeramente en marzo (151), época en que las aves migratorias neárticas inician su viaje de retorno. Mayo (96) y julio (103) son los meses con menor número de especies debido a que están presentes las migratorias australes y las 
migratorias andinas que ocupan los hábitats dejados por las migratorias neárticas (Tabla 2). En setiembre retornan las migratorias neárticas, incrementándose el número de especies a 147, y que se eleva en noviembre a 155, cuando han terminado de llegar la mayor cantidad de especies migratorias neárticas (Fig. 7).

En setiembre se reporta la llegada del mayor número de individuos de Numenius phaeopus, Calidris alba y Charadrius semipalmatus, las del género Tringa y algunas del género Calidris, las cuales también presentan la estadía más prolongada en Pantanos de Villa (García-Olaechea et al., 2018). No obstante, algunas especies de aves que regularmente se encuentran en aguas pelágicas como Phalaropus lobatus y Sterna paradisaea no son vistas regularmente en las playas (Myers, 1983).

Especies como Pluvialis squatarola y Calidris melanotos, fueron raramente observadas registrándose 3 y 23 individuos a lo largo del año; una anotación interesante es que Calidris melanotos siempre fue observado en el mismo lugar (Torres, 2006). El “pato media luna", Anas discors que migra desde América del Norte utiliza los humedales de agua dulce del norte de Colombia y Perú como refugio (Pulido, 2018). Entre las migratorias verdaderamente excepcionales que se registran en la costa del Perú, se tiene a Calidris canutus, Calidris fuscicollis, Tringa solitaria, Stercorarius pomarinus, Sterna maxima y Larus atricilla (Hughes, 1991; Podestá et al., 2017); y Limosa fedoa que durante su permanencia en el Neotrópico, ha sido registrada en humedales de Venezuela, Colombia, Ecuador y Perú; y llega hasta latitudes australes de Chile y Argentina donde culminan su travesía migratoria (González \& Marín, 2017). Para especies como: Calidris canutus, cuyas poblaciones han declinado fuertemente en los últimos años, Limosa haemastica y Calidris fuscicollis, el extremo sur de la Patagonia reviste gran importancia para la conservación de estas especies debido a que constituye una zona de concentración no reproductiva y última parada en la migración de algunas playeras migratorias neárticas de larga distancia (De la Peña, \& Salvador, 2016).

Muy pocos ejemplares de especies migratorias se observan entre junio, julio y agosto. En su mayor parte son principalmente, chorlos y playeros migratorios juveniles e inmaduros que no retornan a sus áreas de reproducción en Norteamérica. Muchos de los individuos de aves migratorias presentes entre octubre y diciembre son individuos inmaduros (Myers, 1983; García-Olaechea et al., 2018). Razones fisiológicas como el ahorro de energía y ecológicas para evitar condiciones climáticas adversas, sugieren su estadía en humedales costeros como Villa, Paracas y lagunas de Mejía (Butler et al., 1996). Algunos sitios específicos son usados como una conducta táctica por individuos de muchas especies migrantes, lo que indicaría que ellos están compitiendo por los recursos dentro de una misma área. En tal sentido la energía y el tiempo invertido en la protección de sus territorios, sugiere a la vez que la defensa de los recursos es fundamental para su supervivencia. Por otra parte los datos históricos de aves anilladas demuestran que las aves migratorias retornan al mismo lugar cada año, quedando además muchos individuos remanentes en el área (Myers et al., 1987). Un estudio sobre chorlos y playeros, indica que las migratorias y residentes se encuentran dentro del espectro de un sistema de explotación, en que cada uno se adapta a determinados recursos específicos (Fuller et al., 2018).

Las migratorias australes que 
proveniente de la Región Antártica, se reproducen en Argentina y Chile y llegan a los humedales de Villa entre abril y agosto (García-Walther et al., 2017). Las especies más características son: Phalaropus lobatus, Chroicocephalus maculipennis, Stercorarius chilensis; Leucophaeus modestus, migra desde la Antártida hasta las costas del Perú, se reproduce en Chile en el desierto de Atacama; y después de anidar, se desplaza hacia el norte a los humedales costeros, Sterna paradisaea, Spheniscus humboldti, Macronectes giganteus, Daption capensis, Pachyptilla belcheri, Puffinus griseus, Oceanites oceanicus, Oceanodroma markhami, Tyrannus savana, el único paserino migrante austral. Gonzalez \& Málaga (1997), registran para la costa de Arequipa, dos especies migratorias del sur de Sudamérica, Muscisaxicola macloviana, entre febrero y noviembre, y Notiochelidon cyanoleuca patagónica, entre mayo y setiembre, las cuales llegan desde las pampas argentinas al Perú.

Algunas especies de aves altoandinas o migrantes verticales emprenden migraciones altitudinales entre los humedales altoandinos hasta las tierras bajas y las áreas costeras durante el invierno Austral, entre abril y agosto. Este patrón definido para Villa, coincide con lo que sucede en las costas de Arequipa (Hughes, 1991) donde las aves provenientes de los Andes se presentan en mayores cantidades entre mayo y noviembre (Gonzalez \& Málaga, 1997), que es la época de sequía en las zonas altas; Phoenicopterus chilensis que llega a la costa durante el invierno; lo mismo que Plegadis ridwayi (González et al., 1999); y Podiceps major migra hacia el norte a lo largo de la costa después de anidar (Delany \& Scott, 2002).

Visitantes ocasionales. Son especies registradas ocasionalmente, algunas de ellas provienen de la Amazonia, otras del comercio ilegal de especies de fauna silvestre de la ciudad de Lima y algunas han sido observadas en años en que se ha producido el fenómeno de "El Niño"; cuya área de distribución no corresponde a los Pantanos de Villa. En total son 31 especies: Nomonyx dominicus, Dendrocigna autumnalis, Sarkidiornis melanotos, Tachibaptus dominicus, Coccyzus melacoryphus, Systellura longirostris, Streptoprocne zonaris, Aramides cajanea, Rallus limicola, Hoploxypterus cayanus, Chlidonias niger, Phaetusa simplex, Pelecanoides garnotii, Mycteria americana, Jabiru mycteria, Fregata magnificens, Sula nebouxii, Sula variegata, Platalea ajaja, Glaucidium brasilianum, Asio flammeus, Caracara plancus, Milvago chimachima, Aratinga erythrogenis, Brotogeris versicolorus, Myiophobus fasciatus, Phaeoprogne tapera, Cyanocorax misticalis, Coereba flaveola, Thraupis palmarum, Saltator striatipectus.

\section{Conclusiones}

Las especies residentes muestran una fuerte filopatría en Pantanos de Villa; los individuos no se dispersan más lejos de su lugar de reproducción. En estas especies la declinación de la población es por causas locales principalmente, la destrucción del hábitat y la contaminación; por ello son las especies residentes las que están sintiendo más fuertemente la pérdida de hábitats.

Los Pantanos de Villa, forman parte de un circuito migratorio mundial de humedales donde las migratorias, particularmente las de larga distancia, son de especial interés porque la destrucción del hábitat está sucediendo a una tasa elelevada en las tierras de invernada de la mayoría de las especies; aunado a los efectos que produce el cambio climático.

Los patrones de migración establecidos indican que hay una sincronización entre ellas. Mientras las residentes están todo el 
año en Pantanos de Villa, las migratorias neárticas llegan en setiembre para volver en marzo a Norteamerica; en abril están llegando las australes y están volviendo en setiembre hacia las tierras de Argentina y Chile; y un patrón que requiere ser definido indica que entre mayo a agosto las migratorias longitudinales se deplazan hacia la costa en las vertientes occidentales de los Andes.

\section{Contribución de los autores}

V.P.: Redacción del texto, metodología de evaluación, ejecución del trabajo de campo, determinación taxonómica de la flora, registro fotográfico; revisión y aprobación del texto final. L.B.: Redacción del texto, metodología de evaluación, ejecución del trabajo de campo, revisión y aprobación del texto final.

\section{Conflictos de intereses}

Los autores declaran no tener conflictos de intereses.

\section{Literatura citada}

Amaro L. \& G. Goyoneche. 2017. Anidación de aves en el Refugio de Vida Silvestre los Pantanos de Villa 2007-2009, Lima-Perú. The Biologist (Lima), 2017, 15(1), jan-jun: 155-171.

Angulo P. F.; T. S. Schulemberg \& E. Puse. 2010. Las aves de los humedales de Eten, Lambayeque, Perú. Ecología Aplicada. 10(1): 71-78.

Bayly, N. J.; K. V. Rosenberg; W. E. Easton; C. Gómez; J. Carlisle; D. N. Ewert; A. Drake \& L. Goodrich. 2018. Major stopover regions and migratory bottlenecks for Nearctic-Neotropical landbirds within the Neotropics: A review. Bird Conservation International (1), 1-26.

Butler, R.; F. Delgado; H. De La Cueva; V. Pulido \& B. Sandercock. 1996. Migration Routes of the Western Sandpiper. Wilson Bull., 108(4): 662-672.

Cruz, Z.; F. Angulo; H. Burger \& R. Borgesa. 2007 Evaluación de aves en la laguna El Paraiso, Lima, Perú. Revista Peruana de Biología 14(1): 139- 144.
De la Peña, M. R. \& S. Salvador. 2016. Aves argentinas: descripción, comportamiento, reproducción y distribución. Charadriidae a Trochilidae. Comunicaciones del Museo Provincial de Ciencias Naturales "Florentino Ameghino" (Nueva Serie) 20, 1-627.

Delany, S. \& D. Scott. Water Bird Population. 2002. Wetlands International, Wageningen, The Netherlands. 226 pp.

Fuller, R. A. \& C. E. Wainwright. 2018. Secrets of intercontinental flight. Nat Ecol Evol. 2(10), 1523-1524. doi: 10.1038/s41559-018-0693-1

Galbraith H.; D. W. Des Rochers; S. Brown \& J. M. Reed. 2014. Predicting Vulnerabilities of North American Shorebirds to Climate Change. PLoS ONE 9(9): e108899. doi:10.1371/journal. pone.0108899.

García Moreno, J.; R. P. Clay \& C. A. Rios Muñoz. 2007. The importance of birds for conservation in the Neotropical region. J. Ornithology 148(2):S321S326.

García Olaechea, A.; C. Chávez Villavicencio, E. TabiIo Valdivieso. 2018. ¿Influyen las aves migratorias neárticas en el patrón estacional de aves de los humedales costeros? Revista Peruana de Biología 25(2): 117 - 122 (Mayo 2018). doi: http://dx.doi. org/10.15381/rpb.v25i2.13281

García Walther, J.; N. R. Senner; H. V. Norambuena \& F. Schmitt. 2017. Atlas de las aves playeras de Chile: Sitios importantes para su conservación. Universidad Santo Tomás. Santiago, Chile. 274 Pp

Garnica, L. 1999. Evaluación ambiental del Fenómeno "El Niño 1997-98" en el Sector Agrario. Revista Peruana de Biología, El Niño 1997-98 y su impacto sobre los ecosistemas marino y terrestre. Eds. J. Tarazona y E. Castillo. 180-182.

Gómez, C.; N. J. Bayly; R. D. Norris; S. A. Mackenzie; K. V. Rosenberg; P. D. Taylor; K. A. Hobson \& C. D. Cadena. 2017. Fuel loads acquired at a stopover site influence the pace of intercontinental migration in a boreal songbird. Sci. Reports 8: 3405.

González. L. G. \& G. Marín. 2017. Primer registro de la aguja moteada (Limosa fedoa) para la isla Margarita. Bol. Inst. Oceanogr. Venezuela, 56(2): 03-07.

González, O. \& E. Málaga. 1997. Distribución de aves en el Valle de Majes, Arequipa, Perú. Ornitología Neotropical (8): 57-69.

González, 0.; A. Tello \& L. Torres. 1999. El Yanavico Plegadis ridwayi de migratorio andino a residente de la costa peruana. Cotinga II: 64-66. 
Hughes, R. 1991. Las aves de la Provincia de Islay. Boletín de Lima 75: 47-54.

lannacone, E. J.; M. Atasi; T. Bocanegra; M. Camacho; A. Montes; S. Santos; H. Zuñiga \& M. Alayo. 2010. Diversity of birds in Pantanos de Villa wetland, Lima, Peru: period 2004-2007. Biota Neotrop. 10 (2): http://www.biotaneotropica.org.br/v10n2/ en/abstract?inventory+bn02610022010.

Koepcke, M. 1964. Las Aves del Departamento de Lima. Edit. M. Koepcke, Lima 118 pp.

McKinnon, E. \& 0. Love. 2018. Ten years tracking the migrations of small landbirds: Lessons learned in the golden age of bio-logging. The Auk 135(4): 834-856.

Morrison, R. I. G \& K. Ross. 1989. Atlas of Neartic Shorebirds on the Coast of South America. Canadian Wildlife Service-Special publication. 324 pp.

Myers, J. P. 1983. Conservation of migrating shorebirds: staging areas, geographic bottlenecks, and regional movements. American Birds 37(1): 2325.

Myers, J. P.; R. I.G. Morrison; P. Z. Antas, B. A. Harrington; T. E. Lovejoy, M. Sallaberry, S. E. Senner \& A. Tarak. 1987. Conservation Strategy for Migratory Species. American Scientist 75: 19-26.

Ocampo Peñuela, N. 2010. El fenómeno de la migración en aves: una mirada desde la Orinoquia. Revista Orinoquia 14(2):188-200.

Osorio Olarte, J. 2012. Aves migratorias neotropicales en parques y jardines de Bogotá: 1945 - 2005. Revista Nodo 6(12): 67-82.

Plenge, M. 2018. List of the birds of Peru / Lista de las aves del Perú. Unión de Ornitólogos del Perú: https:77sites.google.com/site/boletinunop/checklist

Podestá, J.; A. Cotillo; E. Segura Cobeña \& G. CabaniIlas. 2017. Variación temporal de la riqueza y abundancia de aves playeras limícolas en el humedal costero "Poza de la Arenilla"- La Punta, Callao. The Biologist (Lima), 15: 23-35.

Pronaturaleza. 2010. Documento Base para la elaboración de una Estrategia Nacional de Humedales en la Costa Peruana. Fundación Peruana para la Conservación de la Naturaleza. Lima.

Pulido, V. 1987. Patrones de Variación estacional de las poblaciones de aves de las lagunas de Mejía. Tesis para optar el Grado de Magister Scientiae. Universidad Nacional Agraria La Molina. 121 pp.

Pulido, V. 1998. La Zona Reservada de los Pantanos de Villa en el contexto de la conservación de los humedales en el Perú. En: Cano, A. y K. Young (eds.). Los Pantanos de Villa, Biología y Conservación. Museo de Historia Natural - UNMSM. Serie de Divulgación №11: 147-159.

Pulido, V. 2018. Ciento quince años de registros de aves en Pantanos de Villa. Revista Peruana de Biología, 25(3), 291-306. doi: 10.15381/rpb.v25i3.15212.

Pulido, V. \& J. Myers. 1992. Las poblaciones de aves de orilla arenosa marina de Mejía: una propuesta para su conservación. III Congreso Ornitología Neotropical. Cali, 1987.

Pulido, V. \& L. Bermúdez. 2018. Estado actual de la conservación de los hábitats de los Pantanos de Villa, Lima, Perú. Arnaldoa 25(2): 679-702.

Pulido, V.; J. Jahncke; P. Nakamatsu \& C. Flores. 1996. Conservación de los Charadriiformes en la costa peruana. En: Shorebird Ecology and Conservation in the Western Hemisphere. International Wader Studies 8. Presentado al IV Congreso Ornitología Neotropical. 55-61 pp.

Rakhimberdiev, E.; S. Duijns; J. Karagicheva; C. J. Camphuysen; A. Dekinga; R. Dekker \& T. Piersma. 2018. Fuelling conditions at staging sites can mitigate Arctic warming effects in a migratory bird. Nature Communications 9(1), 1-10 doi: 10.1038/ s41467-018-06673-5

Rappole, J. M. 2013. The avian migrant. The biology of bird migration. Columbia University Press, Nueva York. 436 pp. ISBN: 978-0231146784.

Ramsar. 2017. Día mundial de los humedales 2017, Ficha informativa 1: Humedales: una protección natural frente a los desastres. Convención Ramsar, Gland.

Schulenberg, T. S.; D. F. Stotz; D. F. Lane; J. P. O’Neill \& T. A. Parker III. 2010. Aves de Perú. Serie Biodiversidad Corbidi 01. Lima. 660 p.

Schmidt, P.; D. Butler \& D. Petit. 1999. Moving migratory bird management to the next level in North America. Strategies for Conserving Migratory Waterbirds. Wetlands International Publication, Wagening, The Netherlands. (55); 13-16.

Senner, N. R. \& F. Angulo Pratolongo. 2014. Atlas de las Aves Playeras del Perú. Sitios importantes para su conservación. Corbidi. Lima, Perú. 293 pp.

SERNANP. 2016. Prevención, tratamiento y monitoreo de conflictos socioambientales en áreas naturales protegidas de administración nacional. SERNANPSPDA. Lima. 60 pp. 
Torres, M.; Z. Quinteros \& F. Takano. 2006. Variación temporal de la abundancia y diversidad de aves limícolas en el refugio de vida silvestre Pantanos de Villa, Perú. Ecología Aplicada. $\underline{5}$ (1-2): 119-125.

Torres, M. 2006. "Dinámica temporal de la comunidad de aves (Haematopodidae, Charadriidae, Scolopacidae, Recurvirostridae y Phalaropodidae) en la Zona Reservada de los Pantanos de Villa durante el año 2005" Tesis para optar el título de Bióloga. Universidad Nacional Agraria - La Molina.

Torres, M. 2007. (en línea). Evaluación Ornitológica de los Humedales de Puerto Viejo, pantanos de Villa y Humedales de Ventanilla. Serie de publicaciones de flora y fauna silvestre. Instituto Nacional de Recursos Naturales, Lima, Perú. [en línea]. http:// www.inrena.gob.pe/iffs/iffs_biodiv_estud_flora fauna_silvestre.html

Young, K. 1998. El Ecosistema. En: Cano, A. y K. Young (eds.). Los Pantanos de Villa, Biología y Conservación. Museo de Historia Natural - UNMSM. Serie de Divulgación № 11: 3-20. 
TABLA 1. Lista de aves residentes y migratorias por localidad, abundancia y registros, identificadas en los Pantanos de Villa, Lima, Perú.

\begin{tabular}{|c|c|c|c|}
\hline Especie & 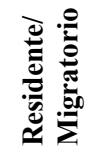 & 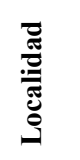 & Nombre común \\
\hline 1. Anas bahamensis & $\mathrm{R}$ & 1 & pato alavanco \\
\hline 2. Anas cyanoptera & $\mathrm{R}$ & 1 & pato colorado \\
\hline 3. Podylimbus podiceps & $\mathrm{R}$ & 1 & zambullidor pico grueso \\
\hline 4. Podiceps major & $\mathrm{R}$ & 1 & zambullidor gigante \\
\hline 5. Rollandia rolland & $\mathrm{R}$ & 1 & zambullidor pimpollo \\
\hline 6. Zenaida asiatica & $\mathrm{R}$ & 1,3 & cuculí \\
\hline 7. Zenaida auriculata & $\mathrm{R}$ & $1,2,3$ & paloma rabiblanca \\
\hline 8. Columba livia & $\mathrm{R}$ & 3 & paloma de Castilla \\
\hline 9. Columbina cruziana & $\mathrm{R}$ & 1,3 & tortolita \\
\hline 10. Crotophaga sulcirostris & $\mathrm{R}$ & 1,3 & guardacaballo \\
\hline 11. Chordeiles acutipennis & $\mathrm{R}$ & 1 & chotacabras trinador \\
\hline 12. Amazilia amazilia & $\mathrm{R}$ & 1,3 & amazilia costeña \\
\hline 13. Thaumastura cora & $\mathrm{R}$ & 1,3 & picaflor de cora \\
\hline 14. Myrtis fanny & $\mathrm{R}$ & 1,3 & picaflor de Fanny \\
\hline 15. Pardirallus sanguinolentus & $\mathrm{R}$ & 1 & gallineta \\
\hline 16. Laterallus jamaicensis & $\mathrm{R}$ & 1 & gallineta oscura \\
\hline 17. Neocrex erythrops & $\mathrm{R}$ & 1 & gallineta pico rojo \\
\hline 18. Gallinula chloropus & $\mathrm{R}$ & 1 & polla de agua \\
\hline 19. Fulica ardesiaca & $\mathrm{R}$ & 1 & gallareta \\
\hline 20. Charadrius alexandrinus & $\mathrm{R}$ & 1,2 & chorlo nevado \\
\hline 21. Charadrius vociferus & $\mathrm{R}$ & 1,2 & chorlo doble collar \\
\hline 22. Haematopus palliatus & $\mathrm{R}$ & 2 & ostrero común \\
\hline 23. Himantopus mexicanus & $\mathrm{R}$ & 1 & perrito \\
\hline 24. Burhinus superciliaris & $\mathrm{R}$ & 1 & huerequeque \\
\hline 25. Ardea alba & $\mathrm{R}$ & 1 & garza blanca grande \\
\hline 26. Egretta caerulea & $\mathrm{R}$ & 1 & garza azul \\
\hline 27. Egretta thula & $\mathrm{R}$ & 1 & garza blanca chica \\
\hline 28. Butorides striatus & $\mathrm{R}$ & 1 & garza tamanquita \\
\hline 29. Bubulcus ibis & $\mathrm{R}$ & 1 & garza bueyera \\
\hline 30. Nycticorax nycticorax & $\mathrm{R}$ & 1 & huaco \\
\hline 31. Nyctanassa violacea & $\mathrm{R}$ & 1 & huaco de corona amarilla \\
\hline 32. Ixobrychus exilis & $\mathrm{R}$ & 1 & garcita leonada \\
\hline 33. Theristicus melanopis & $\mathrm{R}$ & 1 & bandurria \\
\hline 34. Plegadis ridwayi & $\mathrm{R}$ & 1 & yanavico \\
\hline 35. Coragyps atratus & $\mathrm{R}$ & 2,3 & gallinazo cabeza negra \\
\hline 36. Tyto alba & $\mathrm{R}$ & 3 & lechuza de los campanarios \\
\hline 37. Athene cunicularia & $\mathrm{R}$ & 1,2 & lechuza de los arenales \\
\hline 38. Falco sparverius & $\mathrm{R}$ & 1,3 & cernícalo \\
\hline 39. Geositta peruviana & $\mathrm{R}$ & 2 & pampero peruano \\
\hline 40. Phleocryptes melanops & $\mathrm{R}$ & 1 & totorero \\
\hline 41. Camptostoma obsoletum & $\mathrm{R}$ & 2,3 & mosqueta silbadora \\
\hline 42. Tachuris rubrigastra & $\mathrm{R}$ & 1 & siete colores de la totora \\
\hline 43. Pyrocephalus rubinus & $\mathrm{R}$ & 1,3 & turtupilín \\
\hline 44. Lessonia oreas & $\mathrm{R}$ & 1,3 & negrito andino \\
\hline 45. Muscigralla brevicauda & $\mathrm{R}$ & 2 & dormilona cola corta \\
\hline 46. Tyrannus melancholicus & $\mathrm{R}$ & 1,3 & pepite \\
\hline 47. Pygochelidon cyanoleuca & $\mathrm{R}$ & $1,2,3$ & santa rosita \\
\hline 48. Troglodites aedon & $\mathrm{R}$ & 1,3 & cucarachero \\
\hline 49. Mimus longicaudatus & $\mathrm{R}$ & 1,2 & chisco \\
\hline 50. Anthus lutescens & $\mathrm{R}$ & 1 & cachirla amarilla \\
\hline 51. Conirostrum cinereum & $\mathrm{R}$ & 3 & mielerito gris \\
\hline
\end{tabular}


52. Volatinia jacarina

53. Sporophila simplex

54. Sporophila telasco

55. Zonotrichia capensis

56. Agelaius icterocephalus

57. Sturnella bellicosa

58. Molothrus bonariensis

59. Carduelis magellanica

60. Passer domesticus

61. Anas flavirostris

62. Oxyura ferruginea

63. Columbina minuta

64. Metriopelia ceciliae

65. Rhodopis vesper

66. Porphyrula martinica

67. Fulica rufifrons

68. Haematopus ater

69. Sterna lorata

70. Larus belcheri

71. Larus dominicanus

72. Chroicocephalus cirrocephalus

73. Larosterna inca

74. Phalacrocorax bouganvillii

75. Phalacrocorax gaimardi

76. Phalacrocorax brasilianus

77. Pelecanus thagus

78. Pelecanus occidentalis

79. Hydranassa tricolor

80. Cathartes aura

81. Circus cinereus

82. Geranoetus polyosoma

83. Parabuteo unicinctus

84. Heterospizias meridionalis

85. Chloroceryle americana

86. Falco femoralis

87. Aratinga wagleri

88. Forpus coelestis

89. Muscisaxicola macloviana

90. Petrochelidon fulva

91. Petrochelidon rufocollaris

92. Turdus chiguanco

93. Thraupis episcopus

94. Sicalis flaveola

95. Sicalis raimondii

96. Sporophila peruviana

97. Dives warszewiczi

98. Anas discors

99. Chaetura pelagica

100.Porzana carolina

101.Pluvialis dominica

102.Pluvialis squatarola

103. Charadrius semipalmatus

104. Aphriza virgata

105. Arenaria interpres

106. Calidris alba

107. Calidris bairdii
$\mathrm{R}$

$\mathrm{R}$

$\mathrm{R}$

$\mathrm{R}$

$\mathrm{R}$

$\mathrm{R}$

$\mathrm{R}$

$\mathrm{R}$

$\mathrm{R}$

Rn

$\mathrm{Rn}$

$\mathrm{Rn}$

$\mathrm{Rn}$

$\mathrm{Rn}$

$\mathrm{Rn}$

$\mathrm{Rn}$

$\mathrm{Rn}$

$\mathrm{Rn}$

$\mathrm{Rn}$

$\mathrm{Rn}$

$\mathrm{Rn}$

$\mathrm{Rn}$

Rn

$\mathrm{Rn}$

$\mathrm{Rn}$

Rn

Rn

Rn

$\mathrm{Rn}$

Rn

Rn

Rn

Rn

Rn

$\mathrm{Rn}$

Rn

$\mathrm{Rn}$

$\mathrm{Rn}$

$\mathrm{Rn}$

Rn

Rn

$\mathrm{Rn}$

$\mathrm{Rn}$

$\mathrm{Rn}$

$\mathrm{Rn}$

Rn

$\mathrm{Mn}$

Mn

$\mathrm{Mn}$

$\mathrm{Mn}$

$\mathrm{Mn}$

$\mathrm{Mn}$

$\mathrm{Mn}$

Mn

$\mathrm{Mn}$

$\mathrm{Mn}$

1,3

$$
1,2
$$

$$
1,2
$$

$$
\begin{aligned}
& 1,3 \\
& 1
\end{aligned}
$$

$$
1
$$

$$
1,3
$$

1,3

$$
1,3
$$

$$
1
$$

1

$$
3
$$$$
1
$$$$
3
$$$$
\begin{aligned}
& 3 \\
& 1
\end{aligned}
$$$$
1
$$$$
1
$$$$
2
$$$$
2
$$

saltapalito

espiguero simple

espiguero corbatón

gorrión americano

tordo cabeza amarilla

huanchaco

tordo parásito

jilguero cabeza negra

gorrión europeo

pato sutro

pato rana

tortolita menuda

cascabelita

picaflor cola ahorquillada

polla sultana

gallineta frente amarilla

brujilla

gaviotín peruano

gaviota peruana

gaviota dominicana

gaviota capucho gris

zarcillo

guanay

chuita

cushuri

pelícano peruano

pelicano marrón

garza tricolor

gallinazo cabeza roja

gavilán de campo

aguilucho común

gavilán oscuro acanelado

gavilán de sabanas

martín pescador chico

halcón plomado

cotorra cabeza roja

perico esmeralda

dormilona cabeza oscura

golondrina de cuevas

golondrina de collar castaño

chiguanco

violinista celeste

chirigüe

trile bajoandino

espiguero de pico grueso

tordo negro

pato media luna

vencejo de chimenea

gallinetita sora

chorlo dorado

chorlo ártico

chorlo semipalmado

chorlo de las rompientes

vuelvepiedras

playero blanco

playero de Bairdi 
108. Calidris canutus

109. Calidris fuscicollis

110. Calidris himantopus

111. Calidris mauri

112. Calidris melanotos

113. Calidris minutilla

114. Calidris pusilla

115. Calidris ferruginea

116.Limnodromus griseus

117.Limnodromus scolopaceus

118.Tringa flavipes

119. Tringa melanoleuca

120.Tringa solitaria

121. Actitis macularia

122. Catoptrophorus semipalmatus

123. Numenius phaeopus

124. Gallinago gallinago

125.Philomachus pugnax

126. Bartramia longicauda

127.Phalaropus fulicarius

128. Phalaropus tricolor

129. Stercorarius parasiticus

130.Stercorarius pomarinus

131.Leucophaeus atricilla

132.Leucophaeus pipixcan

133. Sterna elegans

134. Sterna hirundinacea

135. Sterna hirundo

136. Sterna maxima

137. Sterna sandvicensis

138.Sterna trudeaui

139. Gelochelidon nilotica

140. Rynchops niger

141. Phoebastria irrorata

142.Pandion haliaethus

143. Buteo platypterus

144.Falco peregrinus

145. Tyrannus tyrannus

146. Progne modesta

147.Progne subis

148. Hirundo rustica

149.Phalaropus lobatus

150.Stercorarius chilensis

151.Leucophaeus modestus

152. Chroicocephalus maculipennis

153. Sterna paradisaea

154.Spheniscus humboldti

155. Macronectes giganteus

156.Daption capensis

157.Pachyptilla belcheri

158. Pufinus griseus

159. Oceanites oceanicus

160. Oceanodroma markhami

161.Tyrannus savana

162.Lophonetta especularoides

163. Anas geórgica

\begin{tabular}{|c|c|c|}
\hline $\mathrm{Mn}$ & 1,2 & playero de Groelandia \\
\hline $\mathrm{Mn}$ & 1,2 & playero lomo blanco \\
\hline $\mathrm{Mn}$ & 1,2 & playero pata larga \\
\hline Mn & 1,2 & playerito occidental \\
\hline $\mathrm{Mn}$ & 1,2 & playero pectoral \\
\hline Mn & 1,2 & playerito pico fino \\
\hline Mn & 1,2 & playerito semipalmado \\
\hline Mn & 1,2 & playero zarapito \\
\hline $\mathrm{Mn}$ & 1,2 & becasina migratoria \\
\hline $\mathrm{Mn}$ & 1,2 & becasina pico largo \\
\hline Mn & 1 & pata amarilla menor \\
\hline Mn & 1 & pata amarilla mayor \\
\hline Mn & 1 & playero solitario \\
\hline Mn & 1 & playero manchado \\
\hline Mn & 1,2 & playero ala blanca \\
\hline Mn & 1,2 & zarapito trinador \\
\hline $\mathrm{Mn}$ & 1 & becasina común \\
\hline Mn & 1 & playero combatiente \\
\hline Mn & 1 & playero de Bartram \\
\hline Mn & 2 & falaropo pico grueso \\
\hline Mn & 1,2 & falaropo de Wilson \\
\hline Mn & 2 & salteador parásito \\
\hline Mn & 2 & salteador pomarino \\
\hline Mn & 1,2 & gaviota reidora \\
\hline $\mathrm{Mn}$ & 1,2 & gaviota de Franklin \\
\hline Mn & 1,2 & gaviotín elegante \\
\hline $\mathrm{Mn}$ & 2 & gaviotín sudamericano \\
\hline Mn & 1,2 & gaviotín común \\
\hline $\mathrm{Mn}$ & 1,2 & gaviotín real \\
\hline Mn & 2 & gaviotín pico punta blanca \\
\hline $\mathrm{Mn}$ & 2 & gaviotín de cabeza blanca \\
\hline $\mathrm{Mn}$ & 2 & gaviotín pico grueso \\
\hline Mn & 1,2 & rayador \\
\hline Mn & 2 & albatros de las Galápagos \\
\hline Mn & 1,2 & águila pescadora \\
\hline Mn & $1,2,3$ & aguilucho ala ancha \\
\hline $\mathrm{Mn}$ & 1,2 & halcón peregrino \\
\hline Mn & 2 & atrapamoscas migratorio \\
\hline $\mathrm{Mn}$ & 3 & golondrina negra \\
\hline Mn & 3 & martín purpureo \\
\hline $\mathrm{Mn}$ & 1,2 & golondrina migratoria \\
\hline $\mathrm{Ma}$ & 1 & falaropo pico fino \\
\hline $\mathrm{Ma}$ & 2 & salteador grande \\
\hline $\mathrm{Ma}$ & 1,2 & gaviota gris \\
\hline $\mathrm{Ma}$ & 1,3 & gaviota capuchicafe \\
\hline $\mathrm{Ma}$ & 2 & gaviotín ártico \\
\hline $\mathrm{Ma}$ & 2 & pingüino de Humboldt \\
\hline Ma & 2 & petrel gigante ártico \\
\hline $\mathrm{Ma}$ & 2 & paloma del cabo \\
\hline $\mathrm{Ma}$ & 2 & petrel azul de pico delgado \\
\hline $\mathrm{Ma}$ & 2 & pardela oscura \\
\hline $\mathrm{Ma}$ & 2 & golondrina de mar de Wilson \\
\hline $\mathrm{Ma}$ & 2 & golondrina de mar Markham \\
\hline $\mathrm{Ma}$ & 1 & atrapamosca cola de tijera \\
\hline Mc & 1 & pato cordillerano \\
\hline Mc & 1 & pato jerga \\
\hline
\end{tabular}


164. Anas puna

165. Chloephaga melanoptera

166.Phoenicopterus chilensis

167.Podiceps occipitalis

168. Apus andecolus

169.Fulica gigantea

170. Recurvirostra andina

171. Thinocorus rumicivorus

172. Chroicocephalus serranus

173. Oceanodroma hornbyi

174. Ardea cocoi

175. Geranoetus melanoleucus

176. Buteogallus meridionalis

177.Progne elegans

178. Riparia riparia

179. Sicalis luteola

180.Dendrocygna autumnalis

181. Sarkidiornis melanotos

182. Nomonyx dominicus

183. Tachybaptus dominicus

184. Coccyzus melacoryphus

185. Systellura longirostris

186. Streptoprocne zonaris

187. Aramides cajanea

188. Rallus limicola

189.|Hoploxypterus cayanus

190. Chlidonias niger

191. Phaetusa simplex

192.Pelecanoides garnotii

193. Mycteria americana

194.Jabiru mycteria

195.Fregata magnificens

196. Sula nebouxii

197. Sula variegata

198. Platalea ajaja

199. Glaucidium brasilianum

200. Asio flammeus

201. Caracara plancus

202. Milvago chimachima

203. Arating a erythrogenis

204. Brotogeris versicolorus

205. Myiophobus fasciatus

206. Cyanocorax mystacalis

207. Phaeoprogne tapera

208. Thraupis palmarum

209. Saltator striatipectus

210. Coereba flaveola

211. Anas platyrhynchos

\begin{tabular}{|c|c|c|}
\hline Mc & 1 & pato puna \\
\hline Mc & 1 & ganso andino \\
\hline Mc & 1 & flamenco \\
\hline Mc & 1 & zambullidor blanquillo \\
\hline Mc & 1,3 & vencejo andino \\
\hline Mc & 1 & gallineta gigante \\
\hline Mc & 1 & avoceta andina \\
\hline Mc & 1,2 & pucopuco menor \\
\hline Mc & 1,2 & gaviota andina \\
\hline Mc & 2 & golondrina de mar acollarada \\
\hline Mc & 1 & garza cuca \\
\hline Mc & $1,2,3$ & aguilucho grande \\
\hline Mc & 1 & gavilán sabanero \\
\hline Mc & 3 & martín sureño \\
\hline Mc & 1,3 & golondrina ribereña \\
\hline Mc & 1,3 & chirigüe común \\
\hline Vo & 1 & pato silbador \\
\hline Vo & 1 & pato arrocero \\
\hline Vo & 1 & pato enmascarado \\
\hline Vo & 1 & zambullidor enano \\
\hline Vo & 3 & cuclillo grisáceo \\
\hline Vo & 1 & chotacabras barba larga \\
\hline Vo & 1 & vencejo collar blanco \\
\hline Vo & 1 & unchala \\
\hline Vo & 1 & gallineta chica \\
\hline Vo & 1 & timelo grande \\
\hline Vo & 2 & gaviotín negro \\
\hline Vo & 2 & gaviotín pico largo \\
\hline Vo & 2 & potoyunco peruano \\
\hline Vo & 1 & manchaco \\
\hline Vo & 1 & jabirú \\
\hline Vo & 2 & tijereta de mar \\
\hline Vo & 2 & camanay \\
\hline Vo & 2 & piquero común \\
\hline Vo & 1 & espátula rosada \\
\hline Vo & 3 & paca paca \\
\hline Vo & 3 & lechuza campestre \\
\hline Vo & 2 & caracara \\
\hline Vo & 1,3 & shihuango blanco \\
\hline Vo & 3 & cotorra frente roja \\
\hline Vo & 3 & pihuicho \\
\hline Vo & 1,3 & mosqueta modesta \\
\hline Vo & 3 & urraca \\
\hline Vo & 1 & golondrina pecho oscuro \\
\hline Vo & 3 & violinista verde \\
\hline Vo & 3 & saltador rayado \\
\hline Vo & 3 & mielerito \\
\hline I & 1 & pato cabeza verde \\
\hline
\end{tabular}




\section{Situación estacional}

$\mathrm{R}=$ Residente de pantanos o mar o áreas arbustivas (60)

$\mathrm{Rn}=$ Residente que no se reproduce en Villa sino en otras áreas de la costa peruana (37)

Vo $=$ Visitante ocasional (31)

Mn = Migratorio del Neártico (51)

Ma = Migratorio Austral (13)

Mc $=$ Migratorio Andes (18)

I = Introducido (1)

\section{Localidad}

1. Pantanos (Totoral, gramadal, espejo de agua).

2. Playa arenosa, mar áreas desérticas.

3. Vegetación arbustiva, jardines, arboledas.

TABLA 2. Estacionalidad bimensual de las especies residentes y provenientes de otras latitudes registradas en los Pantanos de Villa, Lima, Perú.

\begin{tabular}{|c|c|c|c|c|c|c|}
\hline $\begin{array}{c}\text { Residente o } \\
\text { migratorio }\end{array}$ & Enero & Marzo & Mayo & Julio & Setiembre & Noviembre \\
\hline $\mathrm{R}$ & 61 & 61 & 61 & 61 & 61 & 61 \\
\hline $\mathrm{Rn}$ & 36 & 36 & 36 & 36 & 36 & 36 \\
\hline $\mathrm{Mc}$ & 3 & 0 & 18 & 18 & 7 & 5 \\
\hline $\mathrm{Ma}$ & 1 & 1 & 13 & 13 & 3 & 2 \\
\hline $\mathrm{Mn}$ & 51 & 46 & 0 & 0 & 39 & 51 \\
\hline $\mathrm{Vo}$ & 18 & 14 & 0 & 1 & 8 & 11 \\
\hline $\mathrm{I}$ & 1 & 0 & 0 & 0 & 0 & 0 \\
\hline Total & $\mathbf{1 7 1}$ & $\mathbf{1 5 8}$ & $\mathbf{1 2 8}$ & $\mathbf{1 2 9}$ & $\mathbf{1 5 4}$ & $\mathbf{1 7 6}$ \\
\hline
\end{tabular}

$\mathrm{R}=$ Residente

$\mathrm{Rn}=$ Residente que no se reproduce en

Pantanos de Villa

Mc $=$ Migratorio de los Andes

$\mathrm{Ma}=$ Migratorio Austral

Mn = Migratorio Neártico

Vo $=$ Visitante ocasional

$\mathrm{I}=$ Introducido 


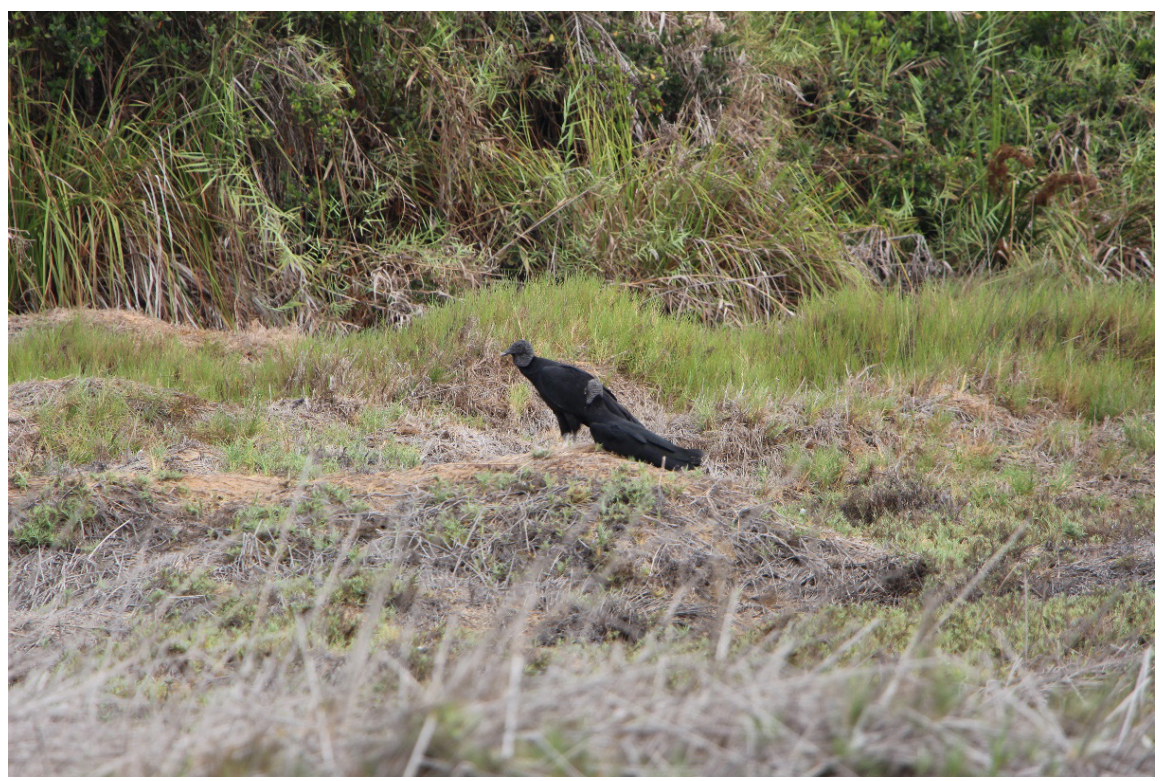

Fig.1. Gallinazo cabeza negra (Coragyps atratus) descansando en hábitat de gramadales.

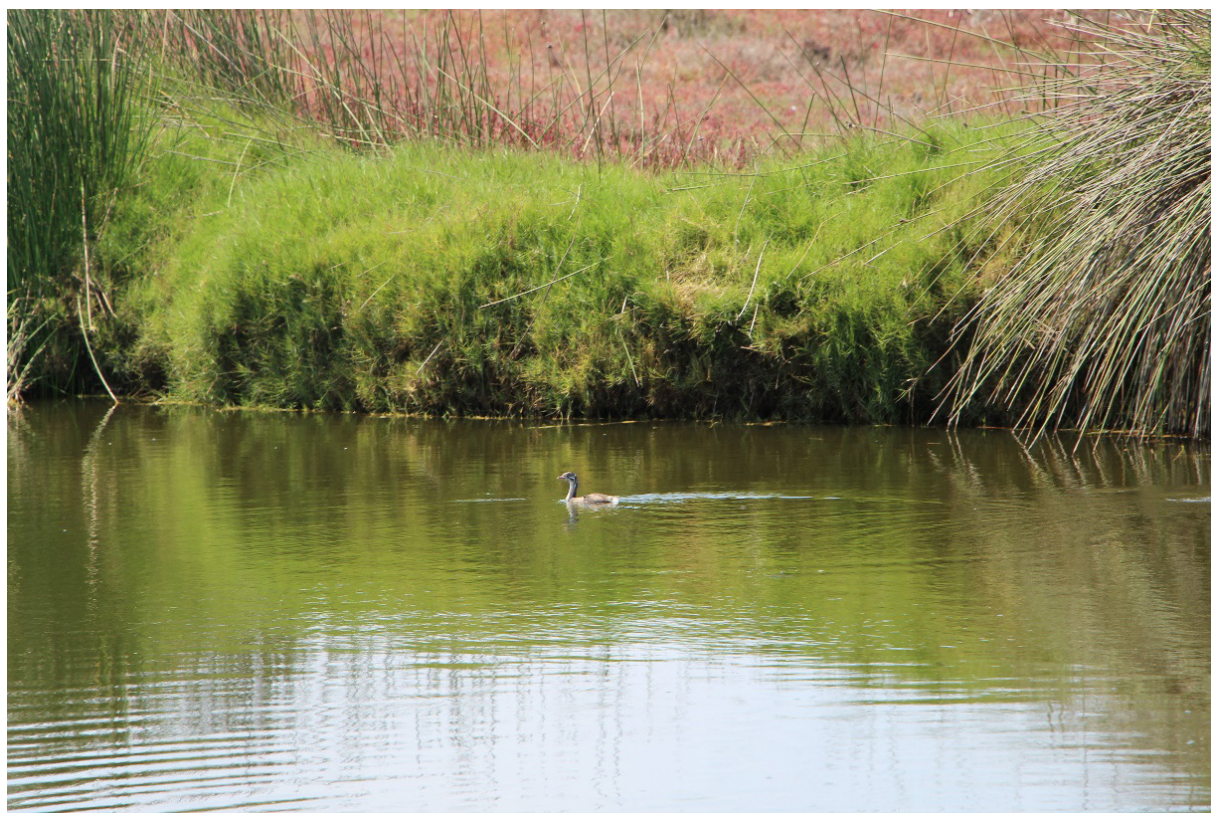

Fig. 2. Zambullidor Rollandia rolland nadando en espejo de agua de las lagunas. 


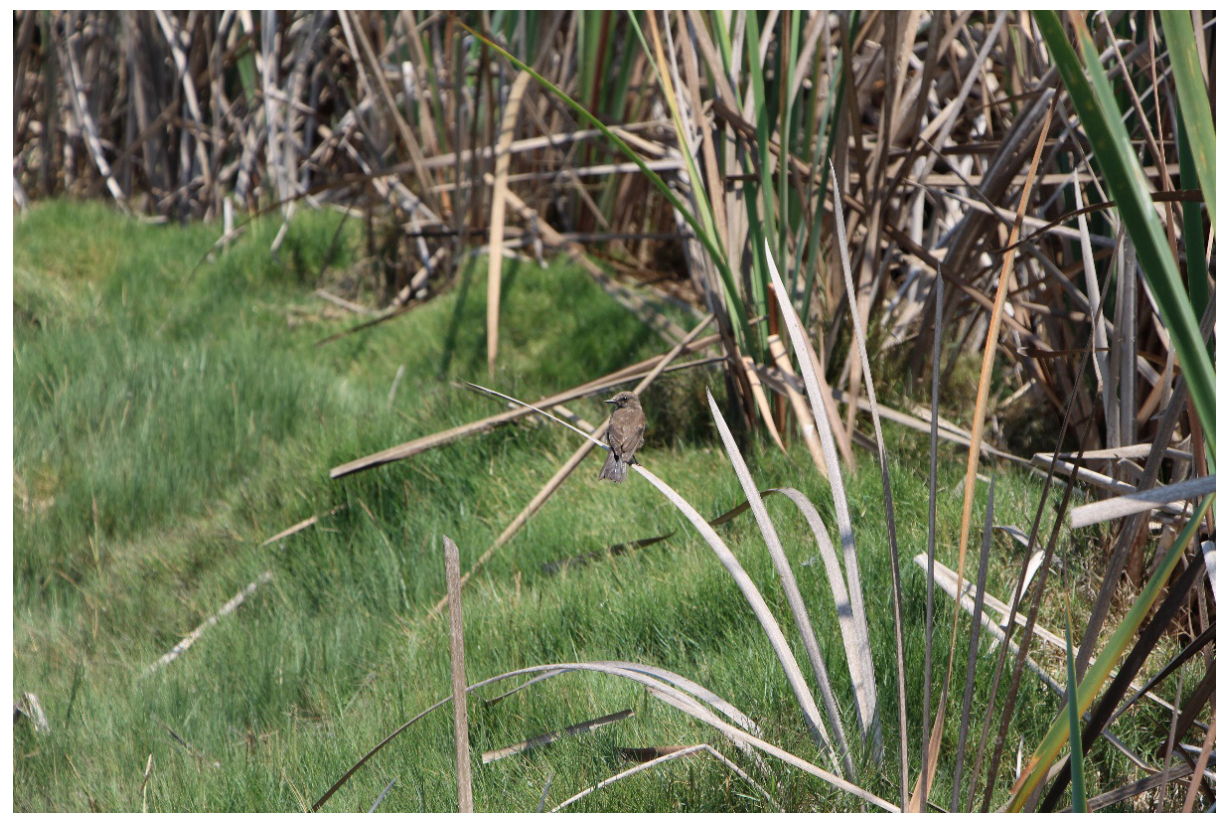

Fig. 3. Turtupilin Pyrocephalus rubinus (fase oscura) sobre totoras.

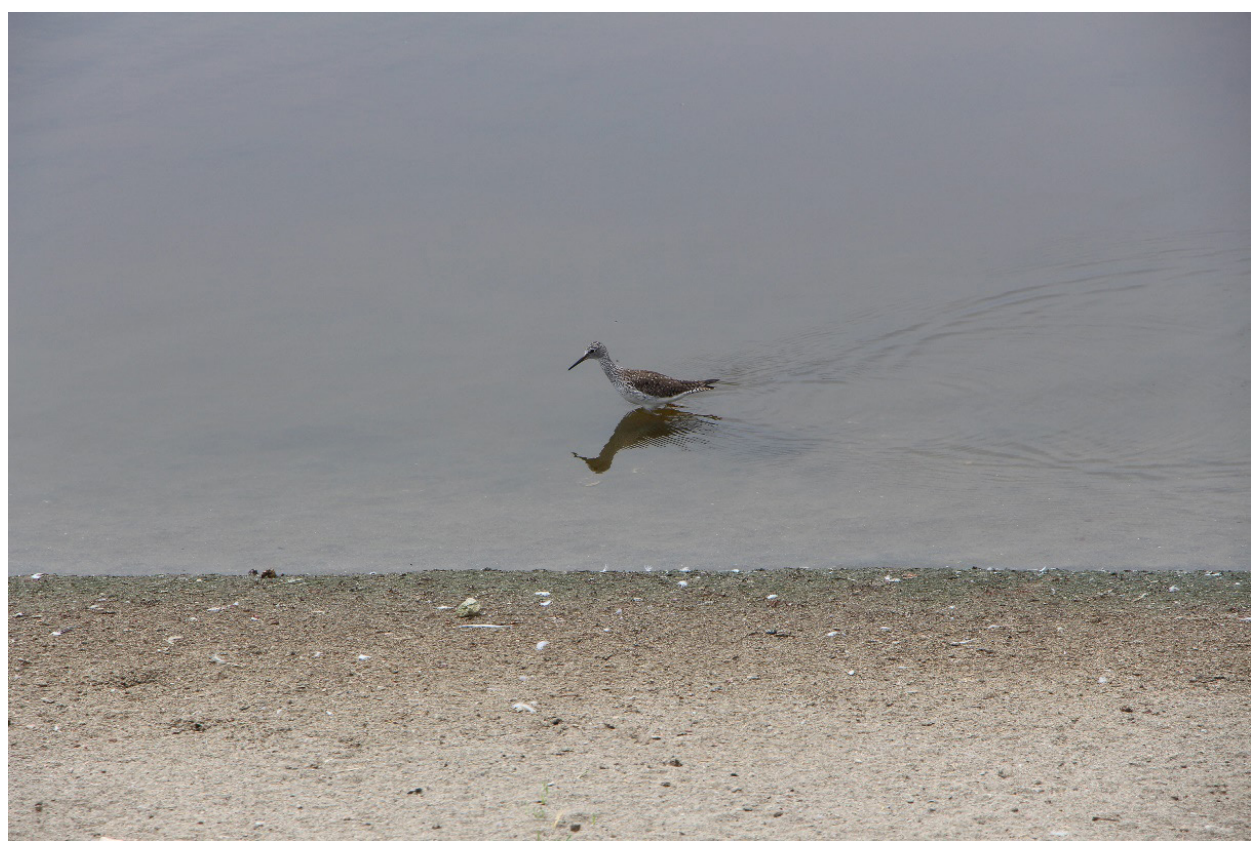

Fig. 4. Pata amarilla Tringa melanoleuca en orilla de espejo de agua. 


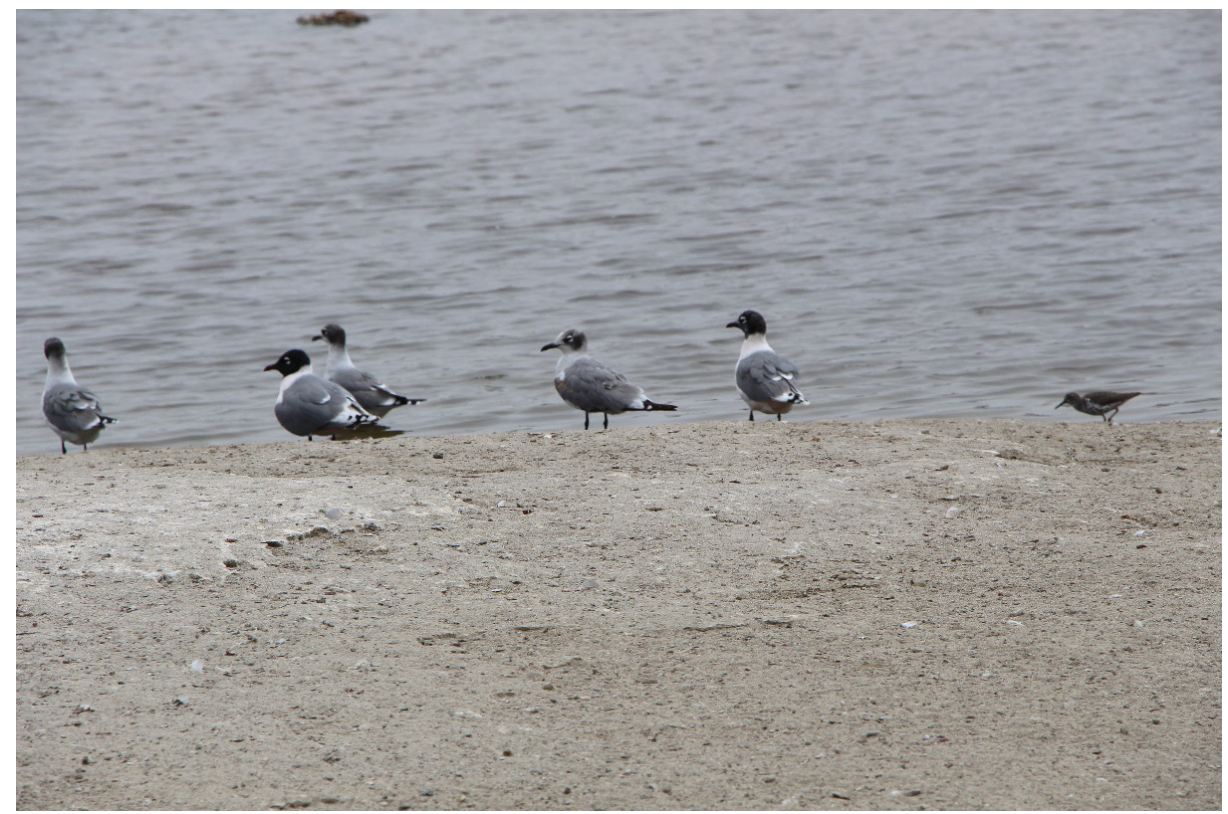

Fig. 5. Gaviota de Franklin Leucophaeus pipixcan, en orilla arenosa.

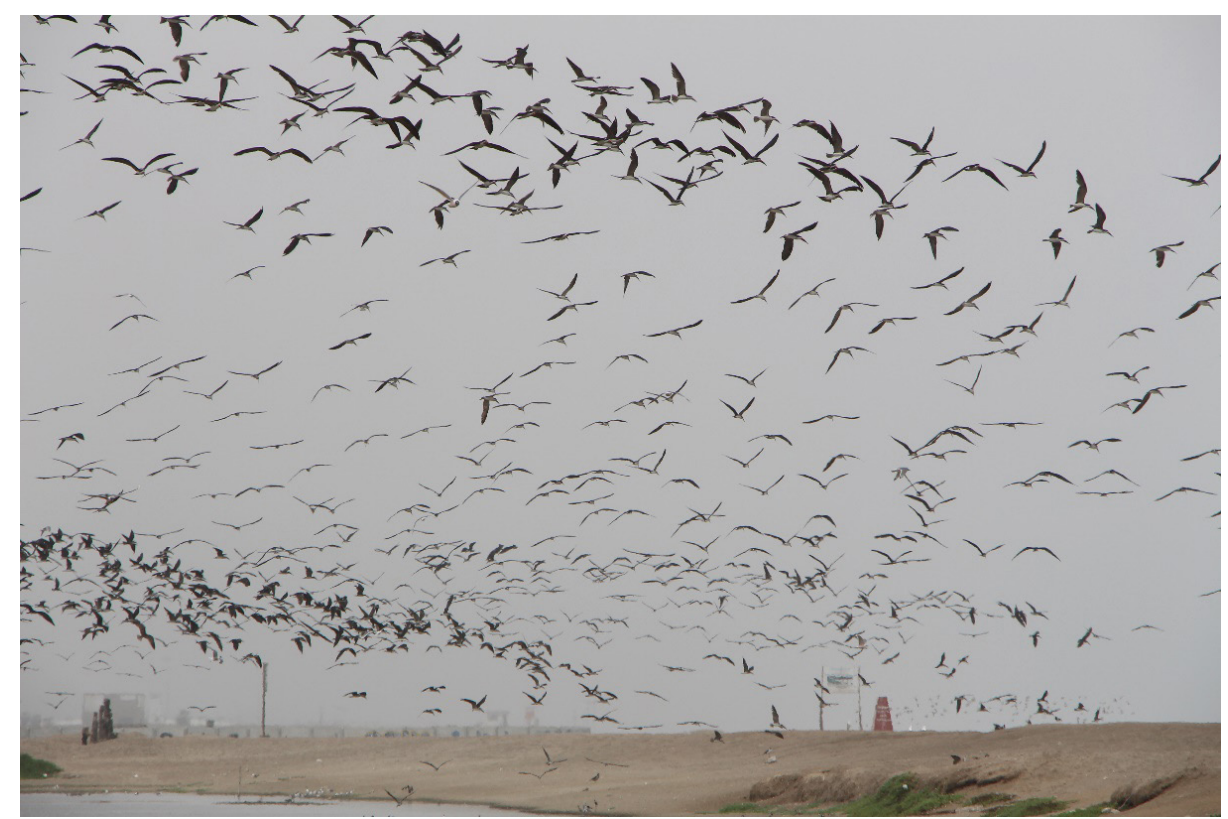

Fig. 6. Bandadas de rayadores Rynchops niger migratoria neártica. 


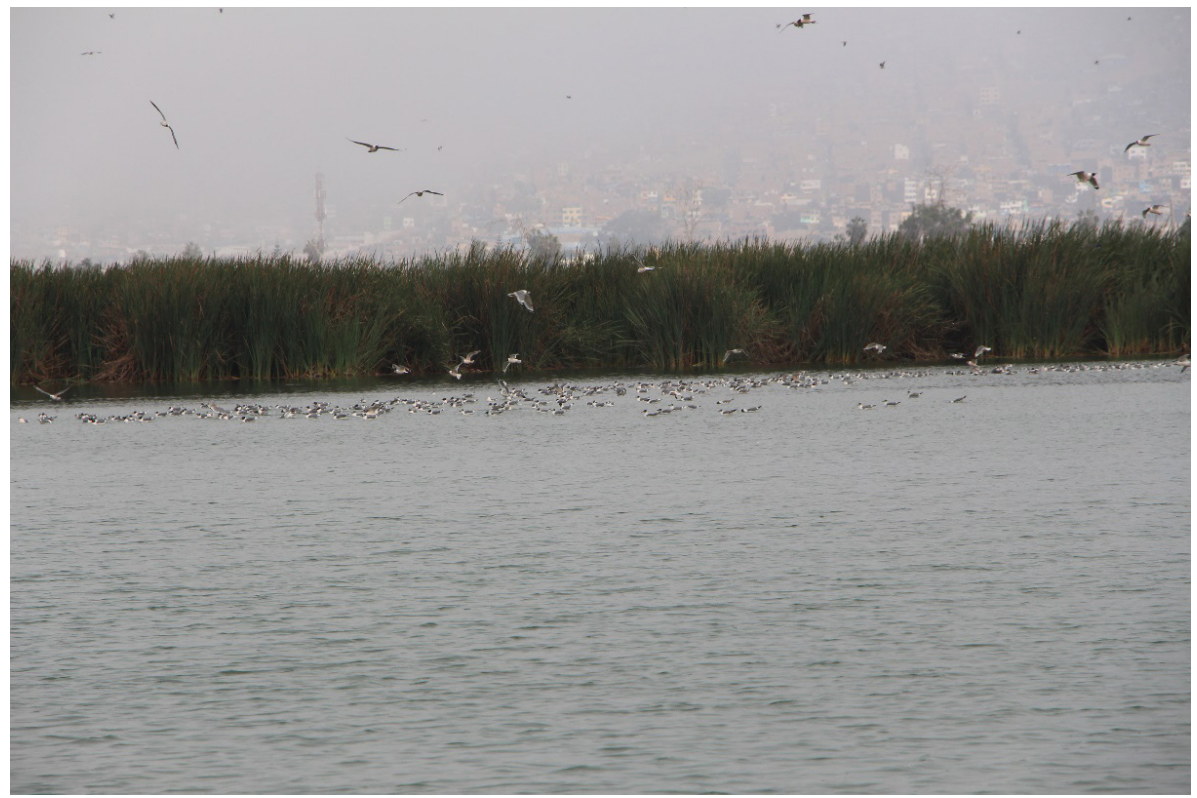

Fig. 7. Bandadas de gaviotas en lagunas de Pantanos de Villa. 
Pulido et al: Patrones de estacionalidad de las especies de aves residentes y migratorias de los Pantanos de Villa, Lima, Perú

1128 I ARNALDOA 25 (3): Setiembre - Diciembre, 2018 